\title{
Measurement of a Structured Backflow in an Open Small Channel Induced by Surface-Tension Gradients
}

\author{
Alba Pulido-Companys, Josep Claret, Jordi Ignés-Mullol, and Francesc Sagués \\ Departament de Química Física, Institut de Nanociència i Nanotecnologia (IN $\left.{ }^{2} U B\right)$, Universitat de Barcelona, \\ Martí i Franquès 1, 08028 Barcelona, Catalonia, Spain \\ (Received 25 January 2013; published 23 May 2013)
}

\begin{abstract}
We present experiments in which the laterally confined flow of a surfactant film driven by controlled surface tension gradients causes the subtended liquid layer to self-organize into an inner upstream microduct surrounded by the downstream flow. The anomalous interfacial flow profiles and the concomitant backflow are a result of the feedback between two-dimensional and three-dimensional microfluidics realized during flow in open microchannels. Bulk and surface particle image velocimetry data combined with an interfacial hydrodynamics model explain the dependence of the observed phenomena on channel geometry.
\end{abstract}

DOI: 10.1103/PhysRevLett.110.214506

Flow control in microfluidic systems has been the subject of intense research in recent years, owing to the potential widespread applicability of miniaturized flow systems in material science, chemistry, and biomedicine [1-5]. Typical experimental arrangements involve narrow pipes carrying fluids into a mixing region where the relevant physicochemical processes take place. Recently, the prospect of designing open-channel alternatives has been explored [6-9] in order to address some of the drawbacks of the standard methods, such as increasing flow resistance and clogging of narrow pipes. Under the confined, low dimensionality conditions of open channel microfluidics, flow control might be exerted through interfacial processes arising at the open interface. For instance, the induction of surface vortex flow through local heating of a phospholipid monolayer could be employed to promote mixing in the underlying subphase, given the coupling between surface and bulk flow [10].

The analysis of laterally bound surfactant monolayer flow along microchannels driven by surface tension gradients has been performed in detail [11,12]. A Poiseuillelike behavior is often observed in monolayers, and the coupling between monolayer and subphase flow determines whether the velocity profile is elliptic or parabolic, depending on the monolayer to subphase viscosity ratio [11]. On the other hand, anomalous flow profiles are reported in the case of viscoelastic surfactant phases [13-16]. In all the performed studies, subphase velocity is simply assumed to decay towards a nonslip condition at the flat bottom of the channel, without much regard to the precise bulk hydrodynamics. Because of mass conservation, continuous monolayer drag should result in a hydrostatic pressure buildup in the subphase downstream, which in turn should trigger a restoring backflow [17]. This process, which might significantly alter the transport patterns in microchannels, both on the monolayer and in the subphase, has been traditionally overlooked in the monolayer flow literature.
PACS numbers: 47.15.gm, 47.55.dk, 47.55.N-, 47.60.Dx

In this Letter, we report on experiments of flow in open microchannels driven by surface tension gradients at an air-water interface decorated with surfactant monolayers. We demonstrate that drag at the interface and lateral confinement determine the formation of backflow patterns that carry the aqueous subphase upstream through self-organized microducts bound by velocity stagnation surfaces. We report surface and bulk particle image velocimetry measurements that, combined with a simple model, allow us to relate the interfacial flow profiles with the shape and location of the stagnation surface. We demonstrate that the observed backflow patterns depend on channel geometry, which can be tuned by adjusting the water level in microfluidic circuits of wettability contrast. The robustness of the reported phenomena is illustrated in a coflow system built on serpentine microchannels.

We control the flow of Langmuir monolayers of the phospholipid dimyristoyl phosphatidylcholine (DMPC [18], Sigma-Aldrich) across a channel that connects two Teflon troughs of dimensions $25 \times 125 \times 3 \mathrm{~mm}^{3}$ (upstream compartment) and $75 \times 125 \times 3 \mathrm{~mm}^{3}$ (downstream compartment), respectively, in a computercontrolled, custom-made assembly [6]. Spring-loaded Delrin barriers are moved independently on both sides of the channel in order to set the surface pressure gradients that drive the interfacial flow. Surface pressure, i.e., surface tension reduction, is monitored by means of electronic Wilhelmy paper-plate balances (Riegler \& Kirstein $\mathrm{GmbH}$ ). The channels are imprinted on a $1.0 \mathrm{~mm}$ thick brass plate as circuits of wettability contrast by means of a combination of photolithographic and wet chemistry methods, as described elsewhere [19]. This process results in hydrophilic tracks surrounded by a superhydrophobic surface. For deeper channels, a groove of known cross section is machined on the brass plate prior to the chemical processing. The shape of the water meniscus standing above the hydrophilic channel is monitored by means of 
structured laser light. For surface velocimetry measurements, the monolayer is doped by $2 \% \mathrm{w} / \mathrm{w}$ of the fluorescent dye Texas-Red DHPE (Invitrogen) and $400 \mathrm{~nm}$ silica nanoparticles (Kisker) are dispersed along with the surfactant after being made hydrophobic through self-assembly coating with $N, N$-dimethyl- $N$-octadecyl3-aminopropyltrimethoxysilyl chloride (DMOAP, SigmaAldrich). For bulk velocimetry measurements, $3 \mu \mathrm{m}$ fluorescent hydrophilic polystyrene particles (micromer red-F-COOH, Micromod $\mathrm{GmbH}$ ) are added to the subphase during monolayer flow experiments. Imaging for velocimetry measurements is performed by means of a custom-built epifluorescence microscope equipped with a high sensitivity digital camera (QImaging Exi-Blue). The instrument is mounted on an $X Y Z$ motorized translation stage. Monolayer control and image acquisition is performed by means of custom-made LABVIEW programs. Image analysis is performed by means of the publicdomain software IMAGEJ [20].

We have studied the monolayer velocity profiles under three different straight channel geometries with a width ranging between 1.6 and $2.0 \mathrm{~mm}$ (see Fig. 1) and a length of $62 \mathrm{~mm}$. For grooved channels, the maximum depth is $0.5 \mathrm{~mm}$ both for round and for rectangular grooves. In the latter case, the monolayer velocity adopts an elliptic profile [see Fig. 1(a)], as expected when subphase drag determines flow resistance. This is true regardless of the height of the water meniscus above the level defined by the surrounding hydrophobic surface. On the other hand, velocity profiles over smooth tracks of wettability contrast reveal an

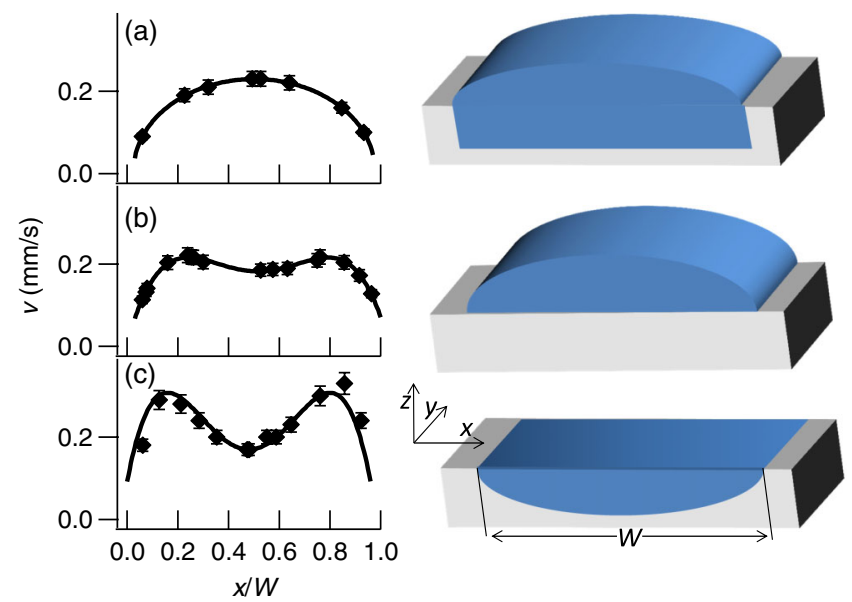

FIG. 1 (color online). Measured velocity profiles across the channel for three different subphase geometries in flow of DMPC monolayers driven by surface pressure gradients (a)-(c). Subphase cross section is shown to the right of each velocity profile. In all cases, water is laterally bound by a superhydrophobic surface. An elliptic flow profile fits the data in (a). Anomalous flow profiles are obtained for geometries (b) and (c). A fourth degree polynomial fit is performed on these data as a guide to the eye and to assist in the analysis of the model detailed in the text. anomalous profile, characterized by a local minimum of the velocity at the center of the channel surrounded by two symmetric maxima [see Fig. 1(b)]. Similar profiles are obtained regardless of the height of the water meniscus, which can be tuned in the range of 0.15 to $0.24 \mathrm{~mm}$ by adjusting the height of the water level in the troughs in contact with the channel plate. The same anomalous profiles can be obtained for the case of a groove with a curved cross section and a flat water meniscus [see Fig. 1(c)]. Increasing the water level results in this case in a progressive change towards the elliptic flow profile.

In all cases, viscous drag with the moving monolayer results in the downstream transport of the subphase, building up a hydrostatic pressure gradient that generates a restoring backflow [17]. We have visualized backflow profiles by dispersing fluorescent hydrophilic microparticles in the subphase. Bulk flow follows the monolayer velocity at the interface, and is damped deeper in the subphase, until a stagnation point (zero velocity) is attained. At higher depths, tracer microparticles move upstream. Although we cannot precisely resolve the vertical location of the particles or the stagnation points, we have obtained a semiquantitative assessment of the backflow distribution by measuring at different positions across the channel the maximum upstream velocity of tracer particles (see Fig. 2 in the main text and Fig. S1 in the Supplemental Material [21]). In order to estimate the dispersion of these data, we have assumed a smooth upstream velocity profile and included a shaded region in each plot with the $99 \%$ confidence interval for the data points. We observe that backflow distribution is correlated with the interfacial flow. Indeed, the former spans almost evenly across the full width of the channel for configurations with an elliptic velocity profile [see Fig. 2(a)] while it is confined inside

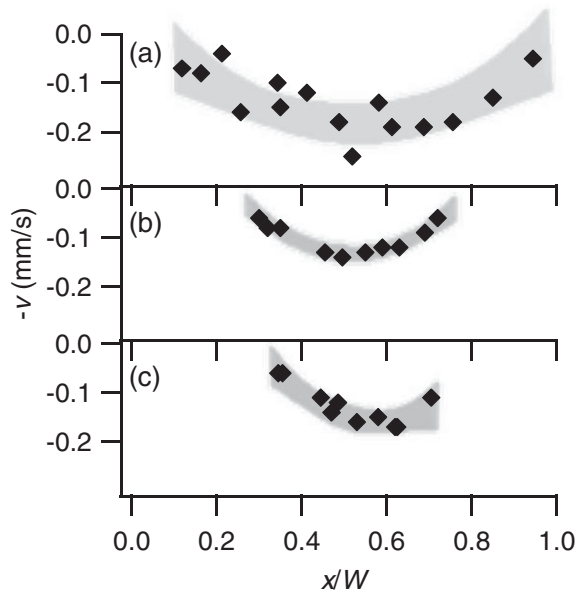

FIG. 2. Measured maximum subphase backflow velocity as a function of the relative position across the channels for the same channel geometries reported in Figs. 1(a)-1(c), respectively. Shaded regions correspond to the $99 \%$ confidence bands of the fit to a second degree polynomial. 
a backflow band for anomalous velocity profiles [see Figs. 2(b) and 2(c)]. In fact, these bands are bound between the points at which the interfacial flow features the two local maxima (see Fig. 1).

The spatial backflow confinement can be qualitatively understood by considering that the subphase upstream flow, which in the steady state should compensate downstream transport caused by drag with the monolayer, follows the path of least resistance. For rectangular grooves and high water level $(0.24 \mathrm{~mm})$, the deepest region in the channel center $(0.75 \mathrm{~mm})$ is just $50 \%$ deeper than the most shallow regions at the channel edges $(0.5 \mathrm{~mm})$. In this case, the backflow band spreads almost evenly across the channel width [see Fig. 2(a)]. On the other hand, for flow over circuits of wettability contrast [see Fig. 1(b)] and over curved grooves with low water levels [see Fig. 1(c)], the depth is almost zero at the channel edges, so subphase backflow resistance will be significantly diminished in the central region, resulting in the self-organized confinement of the backflow.

The observed correlation between interfacial velocity profiles and backflow patterns can be employed to estimate the actual geometry of the backflow duct for a given velocity profile. The monolayer velocity across the channel ( $X$ direction) due to a surface pressure gradient along the channel ( $Y$ direction) satisfies the equation [11]

$$
\mu_{m} \frac{d^{2} v_{m}}{d x^{2}}=\left.\mu \frac{\partial v}{\partial z}\right|_{z=0}+\frac{d \Pi}{d y},
$$

where $v_{m}$ is the monolayer velocity, $v$ is the bulk velocity, $\Pi$ is the surface pressure, $\mu=1 \mathrm{cP}$ is the viscosity of the aqueous subphase, and $\mu_{m} \simeq 10^{-10}-10^{-8} \mathrm{~kg} / \mathrm{s}$ is the shear viscosity of our phospholipid monolayers in the expanded phase [12]. In the thin sublayer limit (channel width larger than subphase depth), the subphase velocity profile can be approximated by a simple shear flow $\partial v /\left.\partial z\right|_{z=0} \simeq v_{m} / H$, where $H$ is the subphase depth [11]. Here, we will regard $H$ as the vertical distance between the interface and the nearest point of zero velocity in the subphase underneath, so that we will be able to estimate $H(x)$ using Eq. (1) after measuring the flow profile across the channel, namely,

$$
H(x)=\frac{\mu v_{m}(x)}{\mu_{m} \frac{d^{2} v_{m}}{d x^{2}}-\frac{d \Pi}{d y}} .
$$

We have computed $H(x)$ for the experimentally measured interfacial velocity profiles $v_{m}(x)$ under different channel geometries (see Fig. 1) and we have obtained the corresponding loci of stagnation by subtracting $H(x)$ to the cross section profile of the water meniscus, obtained from perfilometry measurements. In Eq. (2), $H(x)$ is very sensitive to the value of $d \Pi / d y$, which is too small to be determined with precision using typical surface pressure probes. For this reason, we have resorted to estimating this value so that the computed locus of stagnation has a width consistent with the backflow velocimetry measurements (see Fig. 2). This way, the fitted surface pressure gradients are $1.3 \mathrm{mN} / \mathrm{m}^{2}$ for flow over a smooth substrate, and $0.66 \mathrm{mN} / \mathrm{m}^{2}$ for the curved channel profile [see Figs. 3(b) and 3(c), respectively]. These values correspond to a pressure drop of 0.1 and $0.04 \mathrm{mN} / \mathrm{m}$ along the respective channels, comparable to the $0.1 \mathrm{mN} / \mathrm{m}$ resolution of the surface pressure probe, which prevents a precise direct measurement of the surface pressure drop along the channels. For the rectangular channel profile, the backflow extends across the full width of the channel, which results in an estimation for $d \Pi / d y$ in the range of $0.4-1.6 \mathrm{mN} / \mathrm{m}^{2}$.

In the cases corresponding to the anomalous monolayer velocity profiles, our calculations indicate that backflow is conducted through an immaterial duct, bound by the stagnation region above and by the channel bottom below. Notice that the distance between the interface and the stagnation region is the lowest at the center of the channel [see Figs. 3(b) and 3(c)]. As a result, the vertical gradient of the flow velocity, which partially compensates the driving surface pressure gradient [Eq. (1)] is maximum at the center of the channel, resulting in an increased friction for monolayer flow that leads to the reported anomalous flow profiles featuring a local minimum at the center of the channel.

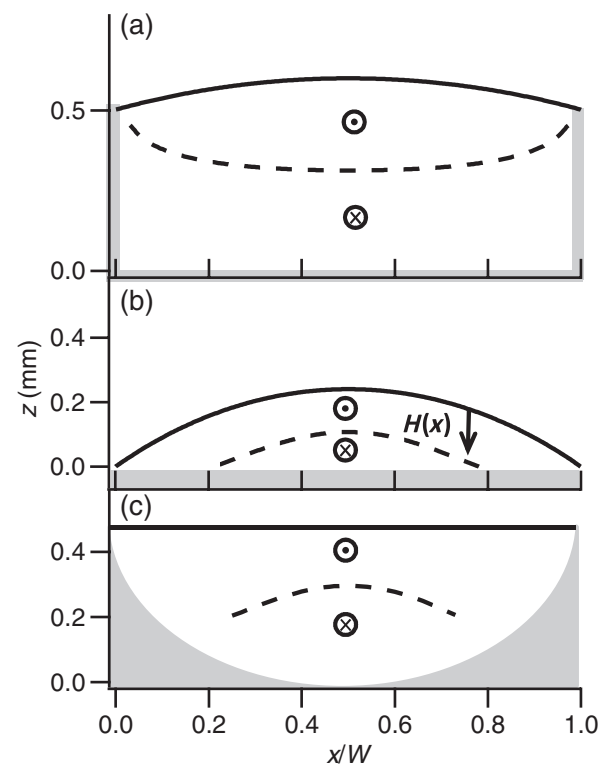

FIG. 3. Cross section with the estimated loci of stagnation confining the region with subphase backflow for the different channel geometries and measured interfacial velocity profiles, corresponding to data in Figs. 1(a)-1(c), respectively, and calculated with Eq. (2). In these diagrams, donwstream flow is pointing towards the reader. Solid lines represent the position of the interface while dashed lines represent the locus of stagnation points. 

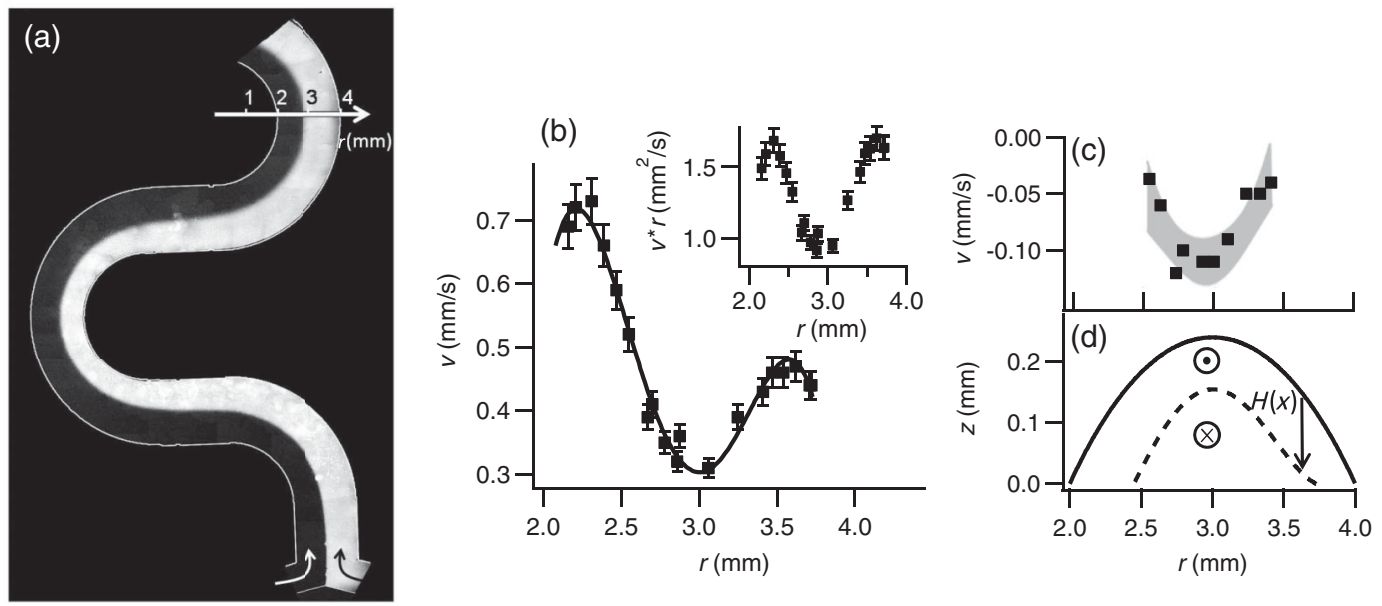

FIG. 4. (a) Interfacial flow and subphase backflow profiles for two monolayers symmetrically coflowing on a serpentine circuit of wettability contrast. The water channel is $2.0 \mathrm{~mm}$ wide. (b) Velocity profile along the arrow segment in (a), and radial scaling (inset). (c) Measured maximum backflow velocity across the channel. (d) Calculated locus of stagnation using Eq. (2) for the flow profile in (a). The solid line represents the position of the interface while the dashed line is the locus of stagnation points.

In order to test the robustness of the described phenomena, we have built a circuit of wettability contrast with serpentine channels where we have studied the coflow of two DMPC monolayers, one of which is doped with fluorescent dye (see Fig. 4). With this arrangement, both interfacial and subphase flow can be measured independently. The flow rates are equal, so the contact line between the coflowing monolayers is located at the center of a rectilinear portion of the channel. The measured interfacial velocity profile adopts a skewed version of the anomalous profile reported above for rectilinear circuits of wettability contrast. Since interfacial flow is driven by the pressure difference between the two ends of the channel, and the trajectory along the curvilinear paths scales with the local radius of curvature, the average velocity of the outer monolayer will be smaller than that of the inner monolayer when flowing along a bend. Evidence for this is the fact that the width of the outer monolayer is higher than that of the inner one on a bend, so that the constraint of equal flow rate is satisfied. Moreover, a robust scaling of the velocity with the radius of curvature is satisfied even for the inhomogeneous flow profile present in this configuration [see the inset in Fig. 4(b)]. We have also measured the maximum upstream backflow velocity at different positions across the channel width [see Fig. 4(c)], and we have calculated the locus of stagnation points that best match the observed backflow profile, as explained above, yielding a surface pressure gradient of $3.6 \mathrm{mN} / \mathrm{m}^{2}$. The asymmetry of the velocity profile is reflected in the backflow pattern, both in the bulk velocimetry measurements and in the calculated locus of stagnation points.

In summary, we have reported experiments on open microfluidic systems in which the confined flow is driven by interfacial pressure gradients. The steady state in this system is the result of a feedback between interfacial and bulk flow patterns, and is characterized by anomalous interfacial flow profiles coupled with the self-organization of the subphase into an immaterial microduct that transports liquid upstream. We have provided a semiquantitative assessment of the three-dimensional flow profile, although a full solution of the hydrodynamic problem that includes the self-sustained backflow is still a pending challenge. Our findings might be employed in open channel microfluidics by dynamically adjusting the self-organized upstream duct, which would be a practical alternative to predesigned chemical channels [9]. On the other hand, tuning the flow profile obtained with a constant interfacial tension gradient by modifying the thickness of the subphase layer can be employed to control the interaction of coflowing monolayers of biomolecules, as demonstrated in Fig. 4.

We thank Thomas M. Fischer for insightful discussions and useful comments. We acknowledge funding from MICINN (Project No. FIS2010-21924-C02-01) and by DURSI (Project No. 2009 SGR 1055). A. P.-C. acknowledges funding from Generalitat de Catalunya through Grant No. 2011-FI-B00131.

[1] H. Song, J. D. Tice, and R. F. Ismagilov, Angew. Chem., Int. Ed. Engl. 42, 768 (2003).

[2] D. J. Beebe, G. A. Mensing, and G. M. Walker, Annu. Rev. Biomed. Eng. 4, 261 (2002).

[3] H. A. Stone, A. D. Stroock, and A. Ajdari, Annu. Rev. Fluid Mech. 36, 381 (2004).

[4] T. Squires and S. Quake, Rev. Mod. Phys. 77, 977 (2005).

[5] P. Tabeling, Introduction to Microfluidics (Oxford University, New York, 2005).

[6] P. Burriel, J. Claret, J. Ignés-Mullol, and F. Sagués, Phys. Rev. Lett. 100, 134503 (2008). 
[7] I. Czolkos, J. K. Hannestad, A. Jesorka, R. Kumar, T. Brown, B. Albinsson, and O. Orwar, Nano Lett. 9, 2482 (2009).

[8] R. Ledesma-Aguilar, R. Nistal, A. Hernández-Machado, and I. Pagonabarraga, Nat. Mater. 10, 367 (2011).

[9] M. Rauscher, S. Dietrich, and J. Koplik, Phys. Rev. Lett. 98, 224504 (2007).

[10] R. Muruganathan, Y. Zhang, and T. M. Fischer, J. Am. Chem. Soc. 128, 3474 (2006).

[11] H. A. Stone, Phys. Fluids 7, 2931 (1995).

[12] C. Barentin, C. Ybert, J.-M. Di Meglio, and J.-F. Joanny, J. Fluid Mech. 397, 331 (1999).

[13] M. L. Kurnaz and D. K. Schwartz, Phys. Rev. E 56, 3378 (1997).

[14] D. J. Olson and G. G. Fuller, J. Non-Newtonian Fluid Mech. 89, 187 (2000).

[15] P. Nghe, S. M. Fielding, P. Tabeling, and A. Ajdari, Phys. Rev. Lett. 104, 248303 (2010).
[16] G. G. Fuller and J. Vermant, Annu. Rev. Chem. Biomol. Eng. 3, 519 (2012).

[17] V. G. Levich, Physicochemical Hydrodynamics, PrenticeHall International Series in the Physical and Chemical Engineering Sciences (Prentice-Hall, Englewood Cliffs, NJ, 1962).

[18] We chose this saturated phospholipid both for its biological relevance and because it features a liquidlike monolayer phase for a wide range of experimental conditions.

[19] P. Burriel, J. Ignés-Mullol, J. Claret, and F. Sagués, Langmuir 26, 4613 (2010).

[20] W. S. Rasband, IMAGEJ (U.S. National Institutes of Health, Bethesda, USA, 1997).

[21] See Supplemental Material at http://link.aps.org/ supplemental/10.1103/PhysRevLett.110.214506 for a realtime video where backflow is visualized during an experiment with geometry (b). 